\title{
Nervous Communication in the Distal and Previous Region of the Arm between the Median and Musculocutaneous Nerves
}

Erika Leide da Silva, Ana Catarina Simonato Serra, Raphael Andrade Oliveira, José Carlos Rainho Netto, Marcelo Calil Burihan and Leandro Nobeschi

Department of Medicine, University of Santo Amaro, São Paulo, São Paulo 04829-300, Brazil

\begin{abstract}
PURPOSE: To describe the nervous communication between median and musculocutaneous nerves. METHODS: 70 upper members of 35 bodies were dissected in the Human Anatomy Laboratory at Santo Amaro University. RESULTS: Only one of the dissected arms has the nervous communication between median and musculocutaneous nerves. CONCLUSION: The communication between median and musculocutaneous nerves, despite already cited in literature, was unusual in our sample, as long as in the dissection of 70 arms only one of them has this communication.
\end{abstract}

Key words: Anatomic variation, nervous communication, median nerve, musculocutaneous nerve.

\section{Introduction}

The nervous communication, anastomosis, between median nerve (MN) and musculocutaneous nerve $(\mathrm{MCN})$, is an event resulting from factors set that occurs in embryogenesis, and not so recurrent. The knowledge of anatomic variations, which engage the nervous anastomosis, is relevant from physical evaluation and surgery practice. The nerve injury causes engines and sensitive deficits in muscles groups and skin areas in a certain way and when nervous anastomosis occur these patterns are not evident as in the normal patterns. In the preoperative, it is necessary to take into account the possibility of nervous routes different from the usual avoiding possible mistakes. The intention of this Project is to describe the nervous communication between $\mathrm{MN}$ and $\mathrm{MCN}$ in the distal arm region in order to help to increase the knowledge about anatomic variations.

\section{Materials and Methods}

70 upper limbs of 35 bodies were dissected in the

Corresponding author: Erika Leide da Silva, research fields: morphology.
Human Anatomy Laboratory at Santo Amaro University (UNISA), Campus 1 . The bodies were kept in a formaldehyde solution and had their forearms dissected in a similar way as in surgery conditions.

\section{Results and Discussion}

From 70 upper limbs dissection were found only one arm (right side) with anastomosis between MCN and $\mathrm{MN}$, located in the distal and anterior region of the upper limb. This finding represents $1.4 \%$ of our entire sample (Fig. 1). We observed that MCN passes through coracobrachialis muscle and in the distal and anterior region of upper limp fuses with MN (Fig. 2).

Nerves are anatomic components from the peripheral nervous system, formed by nervous fibres fibers bundles encircled and sustained by connective tissue cover called endoneurium that covers the nervous fiber, perineurium that covers the nervous fascicle and epineurium that covers the nerve. In epineurium are present the adipose tissue, lymphatic vessels and blood vessels which will fill the nervous fibers and your coating [1]. 


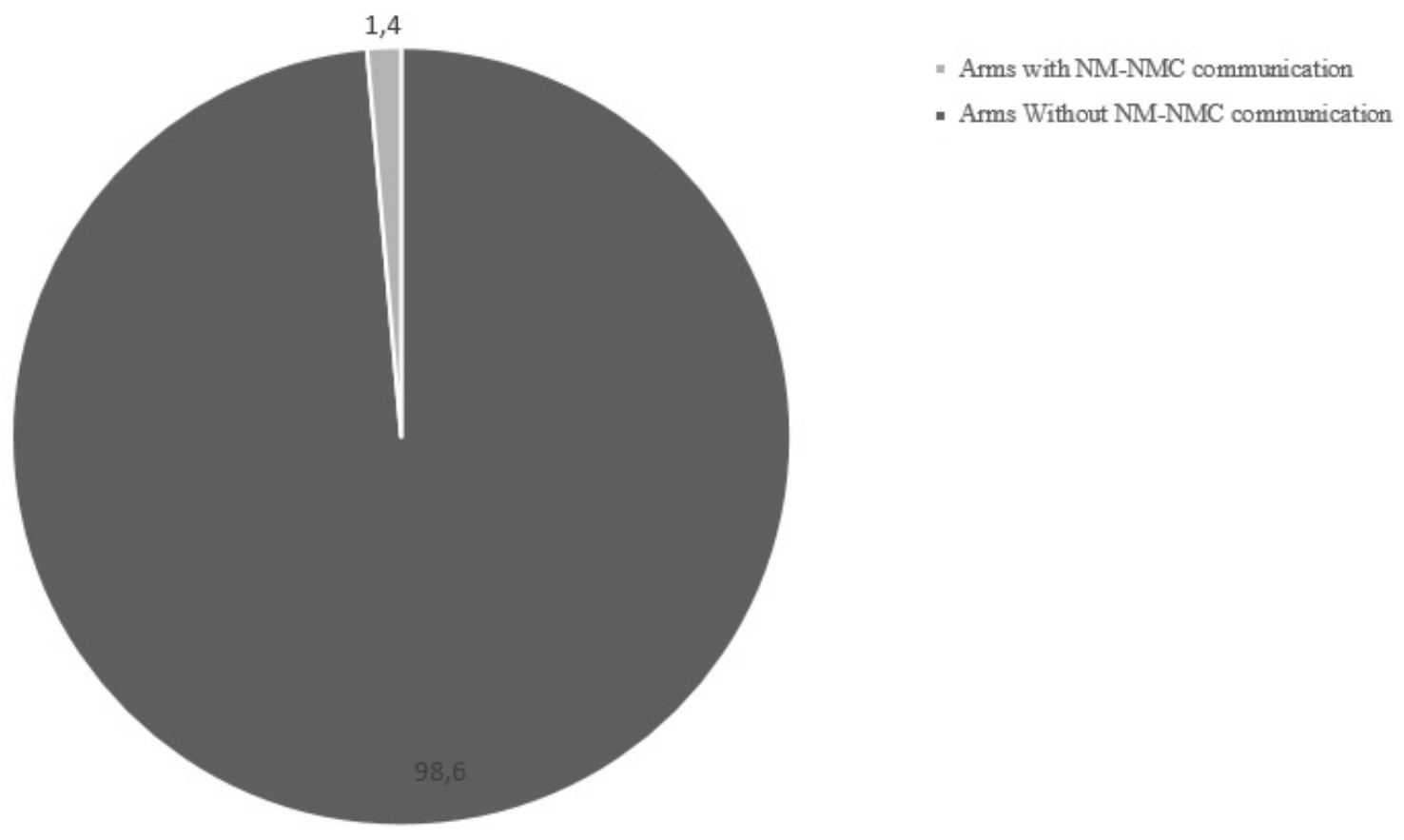

Fig. 1 Frequency of anastomosis between MCN and MN.

* Source: The Human Anatomy Laboratory at Santo Amaro University.

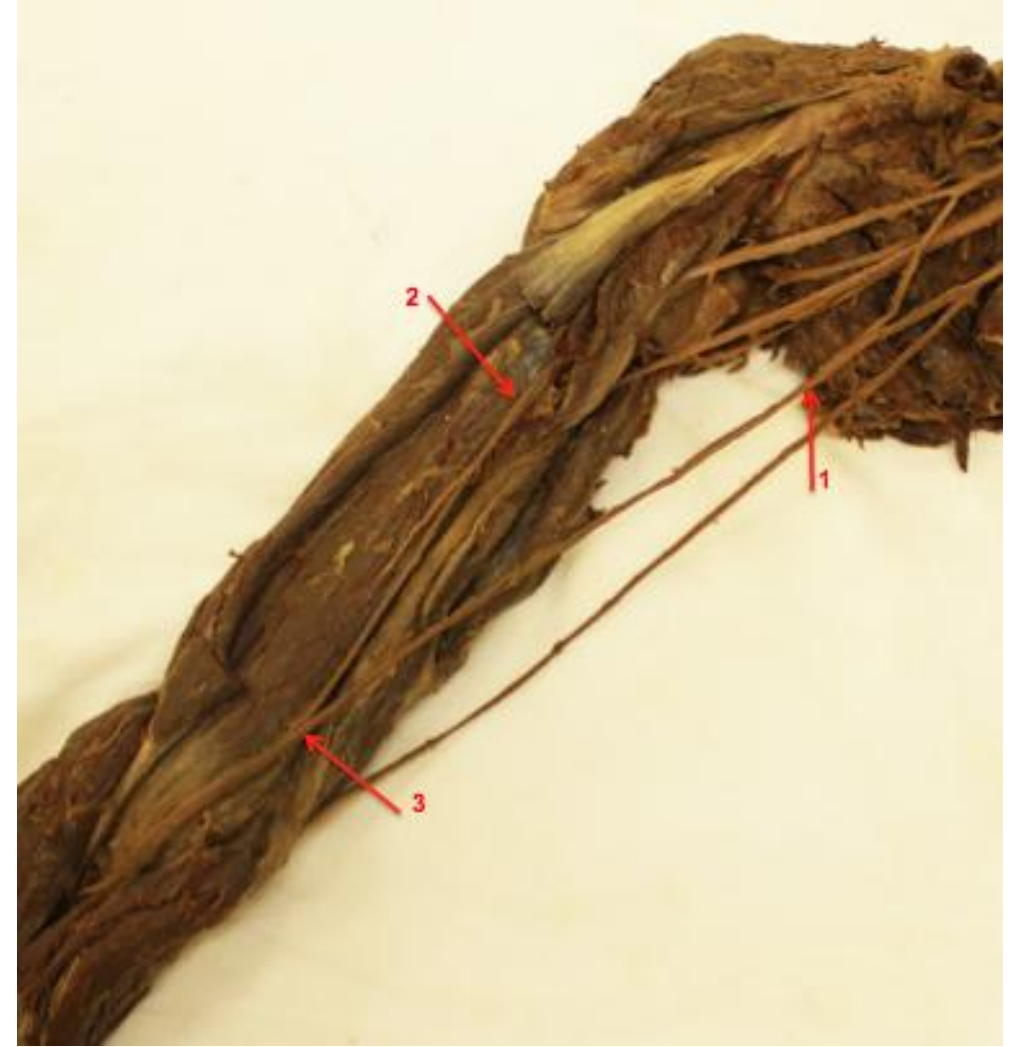

Fig. 2 Previous view from upper limb.

*It's important to look at the MN-formation (1) and its medial path in the upper limb. MCN (2) pierces coracobrachialis muscle and in the distal region communicates with MN (3). 
$\mathrm{MN}$, the main nerve from the anterior compartment of the forearm, derived from the connection of lateral root and lateral fascicle of brachial plexus with the medial root from the medial fascicle. During its route in the forearm, it goes to cubital fossa, medially of the brachial artery, passing through the pronator teres muscle heads. After that, descend in the fascial plane between flexor digitorum superficialis and flexor digitorum profundus, keeping its path in the deep palmaris longus tendon, approaching the flexors muscles retinaculum to cross the carpal tunnel $[1,2]$.

The MN does not innervate arms mucles, however, it sends fibers to supply the forearm musculature (except the flexor carpi ulnaris muscle and flexor digitorum profundus to the 4th and 5th fingers). After crossing the carpal tunnel, it sends fibers to contribute to the pollicis muscle inervation (except the adductor pollicis muscle) and lumbricals hand muscles (1st and 2nd). The cutaneous region of the 1st, $2 \mathrm{nd}$, 3rd, and lateral half of the 4th finger are supplied by the MN [3].

The MCN, which in turn derives from the brachial plexus lateral fascicle, opposite to the bottom margin of the pectoralis minor muscle, situating laterally to $\mathrm{MN}$. It pierces coracobrachialis muscle, innervating this muscle. The MCN continues distally between biceps brachii muscle and brachialis muscle [1, 2]. After supplying the three muscles already cited (owned to anterior compartment of the arm), emerges laterally of biceps brachii muscle called lateral cutaneous nerve of the forearm. MCN-pierces the muscular fascia proximal to cubital fossa, it continues its path with cephalic vein in subcutaneous tissue, becoming subcutaneous to supply the skin of the forearm laterally face [1].

The nerves anastomosis have been considered a congenital variation. The differences in the innervation pattern are results of factors set such as changes in the signal between the mesenchymal cells and growth cones or circulatory factors in the time of branches of the brachial plexus merger or factors that occur during the embryogenesis and can contribute to communication between $\mathrm{MN}$-and MCN [4].

The communication between $\mathrm{MN}$ and $\mathrm{MCN}$ has already been described sometimes in the literature. A study with a body dissection has showed that MCN joins NM after it issues the lateral cutaneous nerve of forearm [5]. However, as already described, this communication happens from MN, which distributes itself in its end in direction to MCN [6]. Communications between $\mathrm{MN}$ and $\mathrm{MCN}$ frequently occurs in the lower third of the arm with a frequency, in this region, of $8 \%$ [7].

Some authors registered the incidence of MN-MCN communication over $40 \%$ of the cases, others, lower than $15 \%[8,9]$. The dissection of 40 arms from 20 fetuses showed that 10 of them had this nervous communication and $90 \%$ of them left from MCN to MN [8].

There are descriptions of this nervous communication adopting as reference the coracobrachialis muscle (when the communication are proximal, distal or doesn't cross the muscle) [4] and pronator teres muscle (when the communication crosses between the two roots, when crosses the ulnar root or when cross under the muscle) [3].

The knowledge about the nervous anastomosis is of major importance to avoid injuries during surgery proceedings and this variation frequently involves the MN-MCN communication through one or more communicating roots $[3-7,8]$.

Differently from resulting of works mentioned above, we found in our study only one case (1.4\%) with MCN-MN communication. This anastomosis occurred in the distal and anterior region of the forearm, with $\mathrm{MCN}$ going to $\mathrm{MN}$, after crossing the coracobrachialis muscle. Clinically, this anastomosis can cause difficulty in the evaluation. An MN injury in the axilla or in the proximal region of the arm would result in a significant decrease of the wrist flexion and hand of benediction. Although, in the presence of this nervous communication, fibers from $\mathrm{MCN}$ go to distal region of the arm, being able to supply the musculature. In this 
case, the classic clinics characteristics from $\mathrm{MN}$ injury would not be observed.

\section{Conclusion}

The MN-MCN communication was a rare finding in our survey, representing $1.4 \%$ of our sample. Our purpose is to report the frequency of this nervous anastomosis, contributing to surgery proceedings and clinical evaluations.

\section{References}

[1] Moore, K. L., Dalley, A. F., and Agur, A. M. R. 2010. Clinically Oriented Anatomy. Philadelphia. Lippincott Williams \& Wilkins. 49-50; 78; 732-4; 756-7.

[2] Paulsen, F., and Waschke, J. 2013. Sobotta-Atlas of Human Anatomy. München. Urban \& Fischer. 131; 194-6; 204-5; 221-3; 226-30; 240-1.

[3] Gambero, P. P. M., Angelini, L. C., De Oliveira B. C., Santos, F. V., Soares, S. A., and Junior, J. G. L. 2012. "Anatomic Variation of Median Nerve in the Elbow-Study in Cadavers." The Anatomist. 4: 25-33. (in Portuguese)

[4] Radunovic, M., Vukasanovic-Bozaric, A., Radojevic N., and Vukadinovic, T. 2013. "A New Anatomical Variation of the Musculocutaneous and the Median Nerve Anastomosis." Folia Morphologica 72 (2): 176-9.

[5] Nascimento, S. R. R., Ruiz, C. R., Pereira, E., Andrades, L., and Souza, C. C. 2016. "Rare Anatomic Variation in the Musculocutaneous Nerve-Case Report." Brasilian Journal of Ortophedic. 51 (3): 366-9. (in Portuguese)

[6] Maeda, S., Kawai, K., Koizumi, M., Ide, J., Tokiyoshi, A., Mizuta, H., and Kodama, K. 2009. "Morphological Study, by Teasing Examination, of the Communication from the Musculocutaneous to Median Nerves." Anatomical Science International 84 (1-2): 41-6.

[7] Guerri-Guttenberg, R. A., and Ingolotti, M. 2009. "Classifying Musculocutaneous Nerve Variations." Clinical Anatomy 22 (6): 671-83.

[8] Caetano, E. B., Vieira, L. A., Cavalheiro, C. S., Filho, M. R., Almargo, M. A. P., and Caetano, M. R. 2016. "Anatomic Study of Nervous Communication between Median and Musculocutaneous Nerves." Brazilian Ortophedic Acta. 24 (4): 200-3. (in Portuguese)

[9] Maeda, S., Kawai, K., Koizumi, M., Ide, J., Tokiyoshi, A., Mizuta, H., and Kodama, K. 2009. "Morphological Study of the Communication between the Musculocutaneous and Median Nerves." Japanese Association of Anatomists 84 (1): $34-40$ 